\title{
Effect of Implementing Discharge Plan on Health Outcomes of the Adult Congestive Heart Failure Patients
}

\author{
Hend I.M. Abd Elaty ${ }^{1}$, Soheir Mostafa Eweda ${ }^{1}$, Thoraya Mohamed Abdel Aziz**1, \\ Samia Mohamed Hafez ${ }^{1}$, Mohamed Ibrahim Sanhoury ${ }^{2}$ \\ ${ }^{1}$ Department of Medical Surgical Nursing, Faculty of Nursing, Alexandria University, Egypt, \\ ${ }^{2}$ Department of Cardiology and Angiology, Faculty of Medicine, Alexandria University, Egypt \\ *Corresponding author: Thoraya Mohamed Abdelaziz, Mobile: (+20) 01222533518, E-Mail: thoraied@yahoo.com
}

\begin{abstract}
Background: Congestive heart failure (CHF) prompt discharge nursing planning is aimed to expand knowledge concerning CHF, promote self-care practices, improve clinical outcomes and minimize readmission.

Objective: Determination of the effect of implementing discharge plan on health outcomes of the adult CHF patients.

Materials and Methods: The study was conducted at the Inpatient Cardiology Department, Alexandria Main University Hospital and its affiliated Outpatient Cardiac Clinic. A convenience sample of seventy hospitalized CHF patients, who were sequentially divided into 2 equal groups; control group: exposed to routine hospital care and study group: received the discharge plan. Four tools were used; Tool I: Congestive Heart Failure Patients' Nursing Assessment, Tool II: Atlanta Heart Failure Knowledge Test, Tool III: Congestive Heart Failure Health Outcome Indicators and Tool IV: Self-Care of Heart Failure Index.

Results: There was significant difference between the two studied groups in relation to overall CHF knowledge mean scores after implementing the discharge plan. A statistical significant difference was noticed between the studied group's patients in relation to overall mean scores of self-care maintenance scale, self-care management scale, and self-care confidence scale.
\end{abstract}

Conclusion: Implementing the developed discharge plan has significantly improved the overall knowledge, selfcare practice confidence of adult CHF patients.

Keywords: Self-Care practice, Health outcomes, Knowledge, Discharge plan, Heart failure, Cardiology nursing.

\section{INTRODUCTION}

Heart failure (HF) is one of the epidemics of modern cardiovascular medicine affecting negatively on patients, their families and global financial resources ${ }^{(\mathbf{1})}$. The number of individuals diagnosed with $\mathrm{CHF}$ is increasing and expected to rise to $46 \%$ by 2030 . However, $1-2 \%$ of the worldwide adult population suffers from $\mathrm{HF}^{(2)}$. It is well known that; CHF is a clinical syndrome leading to the inability of the heart to pump necessary blood volume for meeting the various metabolic demands due to the presence of symptoms of pulmonary or peripheral congestion. Clinical presentations could be acute on top of compensated HF. Its pharmacological treatment is lifelong mandating the patients to follow dietary regimen, monitor body weight, detect fluid retention, and manage changes in energy ${ }^{(3)}$.

Discharge planning is the development of an individualized plan that primarily focuses on improving the quality of discharge patients' compliance, education/counseling, and structured follow-up (4). The clinical cardiology nurse is an integral multidisciplinary team member caring for hospitalized CHF patients applying the nursing process, considering each patient's physical, psychological or spiritual needs ${ }^{(5)}$.

Accordingly; the discharge nursing plan is a continuous process starts early during hospitalization and continues post-discharge; aiming to reduce hospital readmissions, and contributing to clinical stability through improving patients' knowledge and promoting self-care capabilities in order to enhance their self-care maintenance, management, and confidence in managing their condition, which in turn enrich their quality of life (QOL) ${ }^{(6)}$.

Self-care concept includes both the decisions and procedures that should be followed to maintain both healthy life functioning and well-being. Improvement of CHF patients' self-care behavior aimed to clinically practice the non-pharmacological nurse-led management programs ${ }^{(7)}$. These continuous educational nursing interventions are required to improve HF knowledge, encourage selfcare behaviors concerning daily weight monitoring, exercises performance, regular use of medication, as well as monitoring manifestations ${ }^{(8)}$.

The aim of the present work was to; determine the effect of implementing the discharge plan on health outcomes of the adult patients with CHF. It hypothesized an improvement of CHF study group patients' health outcomes in relation to knowledge and self-care scales after the implementation of an individualized nursing discharge plan.

\section{MATERIALS AND METHOD}

Design: A quasi-experimental research design was utilized to fulfill the study aim. 
Subjects: Included $70 \mathrm{CHF}$ patients who were admitted in the inpatient ward and attended for follow-up in Outpatient Clinic of the Department of Cardiology in Alexandria Main University Hospital. They were randomly recruited into two sequential equal groups (35 patients each): Group I: study group received individualized discharge care plan, while Group II: control group received the routine hospital care only.

Inclusion criteria: Adults diagnosed with $\mathrm{CHF}$ for longer than 3 months, are hospitalized on cardiac treatment, able to communicate verbally and did not receive previous CHF discharge plan.

\section{Four tools were utilized:}

Tool one: Congestive Heart Failure Patients' Nursing Assessment Sheet: It was developed after reviewing recent relevant literature ${ }^{(\mathbf{9 - 1 1})}$ and was translated into Arabic by researchers; it consisted of four parts:

Part I: Patient's socio-demographic characteristics, Part II: Patients' health history, Part III: Routine laboratory and diagnostic tests, and Part IV: Patient's health problems/needs.

Tool two: Atlanta Heart Failure Knowledge Test (AHFKT-V2): It was developed and revised by Reilly et $a l .{ }^{(12)}$ and Butts et $a l .{ }^{(13)}$; its reliability = 0.84 and translated into Arabic by researchers. This tool focuses on five CHF knowledge domains; CHF disease process/pathophysiology (2 items), nutrition (11 items), behavior (6 items), medications (6 items), and symptom assessment/management (5 items) with a total of 30 multiple choice questions (MCQ) in order to assess patients' knowledge, health literacy and enable adherence to CHF self-care demands. Each correct answer was converted into 0 to 100 percentages; higher scores indicating adequate knowledge about CHF self-care demands. The knowledge score levels were categorized as good $\leq$ $75 \%-100 \%$, Fair from 50\%-74\% and Poor > 50\%.

Tool three: Congestive Heart Failure Health Outcome Indicators: It was developed after reviewing related literature ${ }^{(\mathbf{1 4 - 1 6})}$ to assess the health outcome indicators after implementing the discharge plan. It consisted of three parts: Part I: Respiratory status indicators included (0-26) items scored as: (0) $=$ Not present, $(1)=$ Deteriorated and $(2)=$ Improved. Part II: Cardiac status indicators comprised (0-20) items scored as: $(0)=$ Not present, (1) $=$ Deteriorated and (2) = Improved. Part III: Functional status indicators contained (0-56) items scored as: $(0)=$ Dependent, $(1)=$ Partial dependent and (2) = Independent. Each part scores were summed to get total health outcome score; higher scores indicates better health outcome indicators.

Tool four: Self-Care of Heart Failure Index (SCHFI V6.2 English): It was developed and updated by Riegel $\boldsymbol{e t}$ al. ${ }^{(17)}$ and Siabani $\boldsymbol{e t}$ al. ${ }^{(18)}$. It composed of 22 questions distributed on three scales: (10) for self-care maintenance, (6) for selfcare management, and (6) for self-care confidence. The three subscales were scored from $(0-100)$ each.

Ethical approval:

An official letter was issued to the Cardiology Department Head after Faculty of Nursing Ethical Committee approval for the study to collect the study data. Privacy was assured. Anonymity and confidentiality were maintained. Patient's right to withdraw at any time was respected.

- An Arabic educational program was developed by the researchers' after reviewing the relevant literature and containing health information regarding $\mathrm{CHF}^{(7,10,12 \text {, and 19). }}$.

- The study tools and the educational program were submitted to 5 experts in Medical-Surgical Nursing field to test their content validity, and necessary modifications were done accordingly.

- Reliability of tools I, II, III and IV was estimated using Cronbach's Alpha test; and were $(0.719,0.84,0.782$ and 0.735 respectively.

- Pilot study was conducted on 7 patients, who were excluded from the study sample, to test tools and the educational program clarity, feasibility, and applicability, and then necessary modification was done.

- The discharge plan was carried-out in four phases:

Phase I: Assessment:

- An individualized interviews were done after patients' admission at Cardiology Department; researchers started by introducing themselves, explaining study aim and obtaining patient's consent.

- Initial both groups assessment was carried out using tool I and II to collect baseline needs, health condition knowledge and its related self-care practices.

- Subjective and objective data were collected through physical examination, to elicit actual and potential clinical problems. Data from control group were collected first.

\section{Phase II: Planning:}

- An individualized discharge care plan was developed for each study group patients based on the assessment phase.

- Steps of patients' discharge plan included:

$\checkmark \quad$ Prioritizing problems and needs.

- Formulating nursing diagnosis based on NANDA-I 2018-2020 $11^{\text {th }}$ editions.

- Setting goals.

- Arranging nursing interventions. 
- Preparing educational booklet learning aid.

\section{Phase III: Implementation (4 sessions):}

- The first session started with initial study group individualized reassessment at the Cardiology Department.

- Second session included application of specific nursing interventions, while using the booklet for providing the mentioned health education items.

- In third session, remaining individualized nursing interventions was implemented, using the developed booklet. While in the fourth session, each hospitalized patient understanding was monitored through a researchers-patient interview for further explanations.

- Each session consumed nearly one hour. Every patient repeated the given instruction at two separate individual sessions. The researchers ensured and enforced patients' use of the distributed educational booklet by the researchers as a reference at home.

Phase IV: Evaluation (3 evaluations):

- First evaluation of both groups was done using tool III 24 hours before discharge.

- Second evaluation was performed one month after discharge, while third evaluation was three months thereafter using tools II and IV at the cardiology outpatient clinic or by phone calls.

- The two groups' tools' scores were compared to determine the discharge plan implementation effect on CHF patients' health outcomes.

- Data were collected from October 2018 to November 2019.

\section{Statistical analysis}

Data were analyzed using IBM SPSS software version 20.0. Qualitative data were described using numbers and percent. Quantitative data were described using range, mean, and standard deviation. The selected level of significance was $\mathrm{P}$ at $5 \%$.

\section{RESULTS}

Table (1) showed socio-demographic data; as more than half of both patients' groups were females (60.0\% , 54.3\% respectively). Both groups' age highest percentages were between 50-60 years (51.4\%, 68.6\% respectively). As regards the educational level, equal percentages of illiterate patients were found in both groups representing $22.8 \%$. No socio-demographics significant difference was declared.

Table (1): Sociodemographic characteristics frequency distribution of the studied groups

\begin{tabular}{|c|c|c|c|c|c|c|}
\hline \multirow{2}{*}{$\begin{array}{l}\text { Patient's socio-demographic } \\
\text { characteristics }\end{array}$} & \multicolumn{2}{|c|}{$\begin{array}{l}\text { Control } \\
(n=35)\end{array}$} & \multicolumn{2}{|c|}{$\begin{array}{c}\text { Study } \\
(\mathbf{n}=\mathbf{3 5})\end{array}$} & \multirow[t]{2}{*}{$\chi^{2}$} & \multirow[t]{2}{*}{$\mathbf{P}$} \\
\hline & No. & $\%$ & No. & $\%$ & & \\
\hline Gender & & & & & \multirow{3}{*}{0.233} & \multirow{3}{*}{0.629} \\
\hline Female & 21 & 60.0 & 19 & 54.3 & & \\
\hline Male & 14 & 40.0 & 16 & 45.7 & & \\
\hline Age & & & & & \multirow{3}{*}{2.143} & \multirow{3}{*}{0.143} \\
\hline $40 \leq 49$ & 17 & 48.6 & 11 & 31.4 & & \\
\hline $50 \leq 60$ & 18 & 51.4 & 24 & 68.6 & & \\
\hline Level of Education & & & & & \multirow{3}{*}{7.029} & \multirow{3}{*}{${ }^{\mathrm{MC}} \mathrm{p}=0.072$} \\
\hline Illiterate & 8 & 22.8 & 8 & 22.8 & & \\
\hline Literate & 27 & 77.2 & 27 & 77.2 & & \\
\hline Marital Status & & & & & \multirow{4}{*}{2.073} & \multirow{4}{*}{${ }^{\mathrm{MC}} \mathrm{p}=0.401$} \\
\hline Married & 29 & 82.8 & 25 & 71.5 & & \\
\hline Widow & 5 & 14.3 & 6 & 17.1 & & \\
\hline Divorced & 1 & 2.9 & 4 & 11.4 & & \\
\hline
\end{tabular}

Table (2) displayed health history frequency distribution between both groups. All patients were complaining of dyspnea, reduced exercise tolerance, and fatigue. Control and study group patients $(80.0 \%, 71.4 \%$ respectively) had IHD. A statistically significant difference between both groups regarding anemia $\left(p=0.031^{*}\right)$ was declared. Approximately three quarters $(74.3 \%)$ of the control group and around half $(51.4 \%)$ of the study group suffered from ischemic cardiomyopathy, with statistically significant difference $\left(\mathrm{p}=0.048^{*}\right)$. Respectively, $37.1 \%$ of control and study groups were diagnosed from $4 \leq 5$ years and from $3 \leq 4$ years. 
Table (2): Frequency distribution of control and study groups' patients according to health history and life style

\begin{tabular}{|c|c|c|c|c|c|c|}
\hline \multirow[t]{2}{*}{ Patients' health history } & \multicolumn{2}{|c|}{$\begin{array}{l}\text { Control } \\
(\mathbf{n}=35)\end{array}$} & \multicolumn{2}{|c|}{$\begin{array}{c}\text { Study } \\
(\mathbf{n}=\mathbf{3 5})\end{array}$} & \multirow[t]{2}{*}{$\chi^{2}$} & \multirow[t]{2}{*}{$\mathbf{P}$} \\
\hline & No. & $\%$ & No. & $\%$ & & \\
\hline \multicolumn{7}{|l|}{ Reasons of hospital admission: } \\
\hline Dyspnea & 35 & 100.0 & 35 & 100.0 & - & - \\
\hline Orthopnea & 23 & 65.7 & 25 & 71.4 & 0.265 & 0.265 \\
\hline Paroxysmal nocturnal dyspnea & 23 & 65.7 & 24 & 70.6 & 0.189 & 0.664 \\
\hline Reduced exercise tolerance & 35 & 100.0 & 35 & 100.0 & - & - \\
\hline Fatigue & 35 & 100.0 & 35 & 100.0 & - & - \\
\hline Nocturnal cough & 14 & 40.0 & 19 & 54.3 & 1.433 & 0.231 \\
\hline Lower limb edema & 34 & 97.1 & 33 & 94.3 & 0.348 & ${ }^{\mathrm{FE}} \mathrm{p}=1.000$ \\
\hline Ascites & 11 & 31.4 & 15 & 42.9 & 0.979 & 0.322 \\
\hline \multicolumn{7}{|l|}{ Associated diseases: } \\
\hline Hyperlipidemia & 12 & 34.3 & 20 & 57.1 & 3.684 & 0.055 \\
\hline Hypertension & 24 & 68.6 & 22 & 62.9 & 0.254 & 0.615 \\
\hline Diabetes mellitus & 21 & 60.0 & 23 & 65.7 & 0.245 & 0.621 \\
\hline Anemia & 14 & 40.0 & 23 & 65.7 & $4.644^{*}$ & $0.031^{*}$ \\
\hline Ischemic heart disease & 28 & 80.0 & 25 & 71.4 & 0.699 & 0.403 \\
\hline \multicolumn{7}{|l|}{ Etiology of heart failure } \\
\hline Hypertrophic cardiomyopathy & 19 & 54.3 & 17 & 48.6 & 0.229 & 0.632 \\
\hline Ischemic cardiomyopathy & 26 & 74.3 & 18 & 51.4 & $3.916^{*}$ & $0.048^{*}$ \\
\hline Valvular heart disease & 25 & 71.4 & 22 & 62.8 & 1.701 & 0.192 \\
\hline Anemia & 6 & 17.1 & 3 & 8.6 & 1.148 & ${ }^{\mathrm{FE}} \mathrm{p}=0.477$ \\
\hline \multicolumn{7}{|l|}{$\begin{array}{l}\text { Years of being diagnosed with heart } \\
\text { failure }\end{array}$} \\
\hline $1 \leq 4 \mathrm{yrs}$ & 18 & 51.4 & 20 & 57.1 & \multirow{2}{*}{0.740} & \multirow{2}{*}{${ }^{\mathrm{MC}} \mathrm{p}=0.893$} \\
\hline 4 More than 5 yrs & 17 & 48.6 & 15 & 42.9 & & \\
\hline \multicolumn{7}{|l|}{ Patients' life style } \\
\hline \multicolumn{7}{|l|}{ Smoking } \\
\hline No & 20 & 57.1 & 18 & 51.4 & \multirow{2}{*}{0.230} & \multirow{2}{*}{0.631} \\
\hline Yes & 15 & 42.9 & 17 & 48.6 & & \\
\hline \multicolumn{5}{|l|}{$\begin{array}{l}\text { Dietary intake: } \\
\text { Likes }\end{array}$} & \multirow[t]{2}{*}{ - } & \multirow[t]{2}{*}{-} \\
\hline Not consistent with recommended diet & 35 & 100.0 & 35 & 100.0 & & \\
\hline \multicolumn{7}{|l|}{ Dislikes } \\
\hline Not consistent with recommended diet & 28 & 80.0 & 31 & 88.6 & - & - \\
\hline
\end{tabular}

FE: Fisher Exact

Table (3) illustrated patients' clinical data, where chemistry tests, $\mathrm{CBC}$, and echocardiography examination were requested for all patients. Concerning CHF nursing diagnosis; excess fluid volume, activity intolerance and knowledge deficit were observed in $100 \%$ of all patients. 
Table (3): Frequency distribution of control and study groups' patients according to clinical data

\begin{tabular}{|c|c|c|c|c|c|c|}
\hline \multirow[t]{2}{*}{ Patients' clinical data } & \multicolumn{2}{|c|}{$\begin{array}{l}\text { Control } \\
(\mathbf{n}=35)\end{array}$} & \multicolumn{2}{|c|}{$\begin{array}{c}\text { Study } \\
(\mathrm{n}=35)\end{array}$} & \multirow[t]{2}{*}{$\chi^{2}$} & \multirow[t]{2}{*}{$\mathbf{p}$} \\
\hline & No. & $\%$ & No. & $\%$ & & \\
\hline \begin{tabular}{|l} 
Biochemical Tests: \\
CBC \\
Chemistry tests \\
Coagulation tests \\
\end{tabular} & $\begin{array}{l}35 \\
35 \\
19 \\
\end{array}$ & $\begin{array}{c}100.0 \\
100.0 \\
54.3 \\
\end{array}$ & $\begin{array}{l}35 \\
35 \\
17 \\
\end{array}$ & $\begin{array}{c}100.0 \\
100.0 \\
48.6 \\
\end{array}$ & $\begin{array}{c}- \\
- \\
0.229 \\
\end{array}$ & $\begin{array}{c}- \\
- \\
0.632 \\
\end{array}$ \\
\hline \begin{tabular}{|l|} 
Diagnostic Tests: \\
Chest X-ray \\
Ultrasound abdomen \\
Echocardiography \\
\end{tabular} & $\begin{array}{l}29 \\
10 \\
35 \\
\end{array}$ & $\begin{array}{c}82.9 \\
28.6 \\
100.0 \\
\end{array}$ & $\begin{array}{l}27 \\
12 \\
35 \\
\end{array}$ & $\begin{array}{c}77.1 \\
34.3 \\
100.0 \\
\end{array}$ & $\begin{array}{c}0.357 \\
0.265 \\
-\quad \\
\end{array}$ & $\begin{array}{c}0.550 \\
0.607 \\
- \\
\end{array}$ \\
\hline \begin{tabular}{|l|} 
Nursing diagnosis: \\
Reduced cardiac output \\
Excess fluid volume \\
Activity intolerance \\
Knowledge deficit \\
Risk for impaired gas exchange \\
Anxiety \\
\end{tabular} & $\begin{array}{l}35 \\
35 \\
35 \\
35 \\
20 \\
18 \\
\end{array}$ & $\begin{array}{c}100.0 \\
100.0 \\
100.0 \\
100.0 \\
57.1 \\
51.4 \\
\end{array}$ & $\begin{array}{l}35 \\
35 \\
35 \\
35 \\
20 \\
24 \\
\end{array}$ & $\begin{array}{c}100.0 \\
100.0 \\
100.0 \\
100.0 \\
57.1 \\
68.6 \\
\end{array}$ & $\begin{array}{c}- \\
- \\
- \\
- \\
0.000 \\
2.143 \\
\end{array}$ & $\begin{array}{c}- \\
- \\
- \\
- \\
1.000 \\
0.143 \\
\end{array}$ \\
\hline
\end{tabular}

Table (4) displayed the overall knowledge mean percent scores after one and three months, where they were improved significantly in the study group representing $94.48 \% \pm 15.55 \%$ and $93.05 \% \pm 17.55 \%$ respectively compared to constant mean percent scores $(18.95 \% \pm 6.90 \%)$ in control group. There was statistically significant difference $\left(\mathrm{P}<0.001^{*}\right)$ in relation to overall CHF knowledge mean scores.

Table (4): Overall knowledge mean scores of control and study CHF patients during hospitalization, after one month and 3 months post implementing the discharge plan

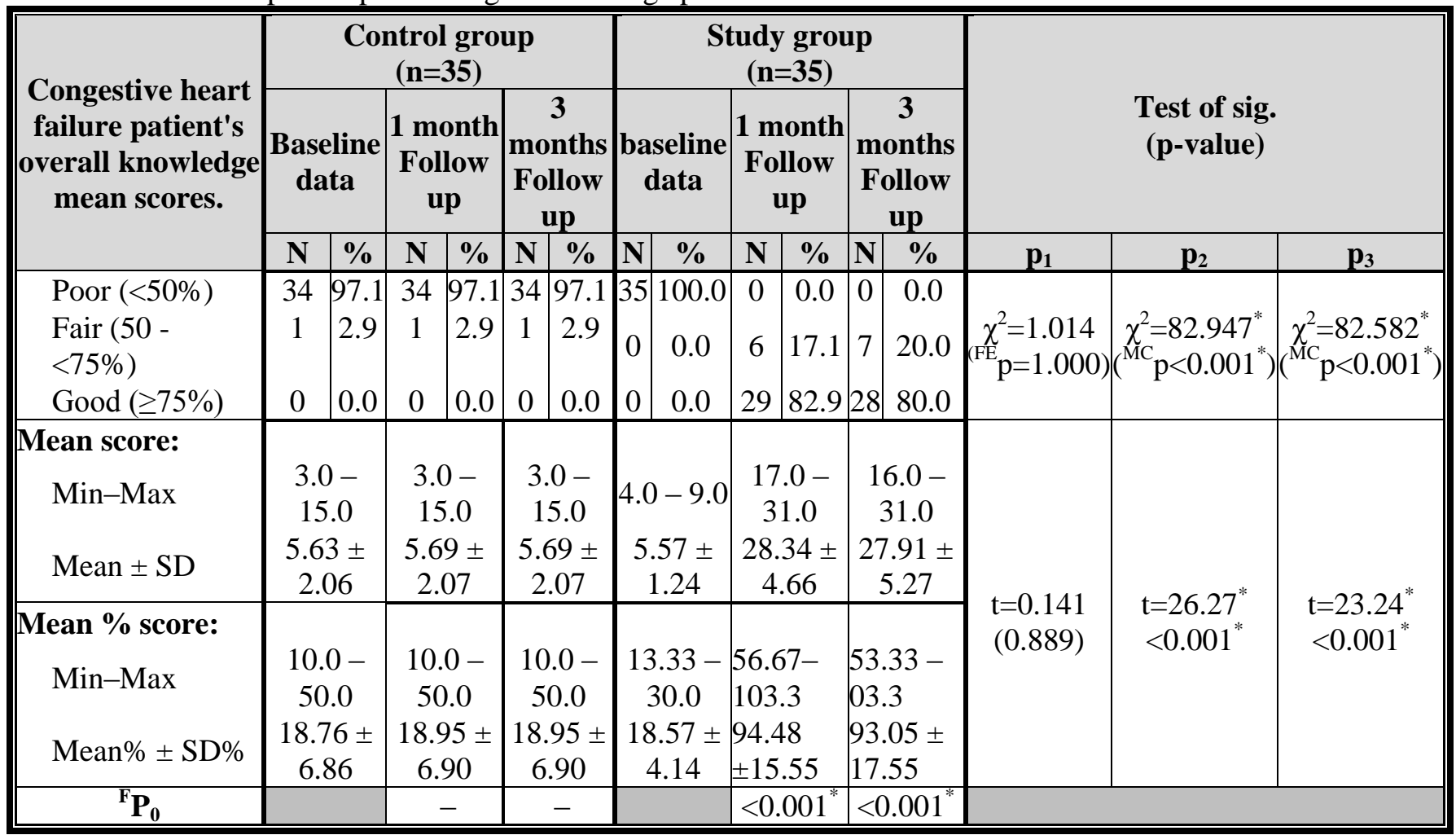

t: Student t-test $\quad$ F:F-test $\mathrm{p}$ : $\mathrm{p}$ value for comparing between the studied periods in each group $\mathrm{p}_{1}$ : $\mathrm{p}$ value for comparing between the studied groups in Baseline, $p_{2}: p$ value for comparing between the studied groups in $\mathbf{1}$ month follow up (F/U), $p_{3}: p$ value for comparing between the studied groups in $\mathbf{3}$ month follow up (F/U), *: Statistically significant at $\mathrm{p} \leq 0.05$

Furthermore, overall congestive heart failure patients' health status indicators 24 hours before hospital discharge, mean scores were $92.83 \pm 3.14$, and $91.43 \pm 4.56$ respectively among control and study groups patients' with no statistically significant difference between the two studied groups $(\mathrm{p}=0.139)$ as shown in table (5). 
Table (5): Comparison between studied groups related to their health outcome indicators 24 hours before hospital discharge

\begin{tabular}{|c|c|c|c|c|c|c|c|c|c|c|c|c|c|c|}
\hline \multirow{3}{*}{$\begin{array}{c}\text { Health } \\
\text { outcome } \\
\text { indicators. }\end{array}$} & \multicolumn{6}{|c|}{ Control group $(\mathrm{N}=35)$} & \multicolumn{6}{|c|}{ Study group $(\mathrm{N}=35)$} & \multirow{3}{*}{$\chi^{2}$} & \multirow{3}{*}{$\mathbf{p}$} \\
\hline & \multicolumn{2}{|c|}{ Independent } & \multicolumn{2}{|c|}{$\begin{array}{c}\text { Partial } \\
\text { dependent }\end{array}$} & \multicolumn{2}{|c|}{ Dependent } & \multicolumn{2}{|c|}{ Independent } & \multicolumn{2}{|c|}{$\begin{array}{c}\text { Partial } \\
\text { Dependent }\end{array}$} & \multicolumn{2}{|c|}{ Dependent } & & \\
\hline & $\mathbf{N}$ & $\%$ & $\mathbf{N}$ & $\%$ & $\mathbf{N}$ & $\%$ & $\mathbf{N}$ & $\%$ & $\mathbf{N}$ & $\%$ & $\mathbf{N}$ & $\%$ & & \\
\hline $\begin{array}{l}\text { Part I: Overall } \\
\text { respiratory } \\
\text { status indicators } \\
\text { mean scores: }\end{array}$ & \multicolumn{6}{|c|}{$21.51 \pm 1.77$} & \multicolumn{6}{|c|}{$20.66 \pm 2.63$} & $\mathbf{t}=\mathbf{1 . 5 9 7}$ & 0.115 \\
\hline $\begin{array}{l}\text { Part II: Overall } \\
\text { cardiac status } \\
\text { indicators mean } \\
\text { scores: }\end{array}$ & \multicolumn{6}{|c|}{$18.23 \pm 0.94$} & \multicolumn{6}{|c|}{$18.17 \pm 0.57$} & $\mathbf{t}=\mathbf{0 . 3 0 7}$ & 0.760 \\
\hline $\begin{array}{l}\text { Part III: Overal } \\
\text { functional status } \\
\text { indicators mean } \\
\text { scores: }\end{array}$ & \multicolumn{6}{|c|}{$53.09 \pm 2.94$} & \multicolumn{6}{|c|}{$52.60 \pm 3.50$} & $t=0.628$ & 0.532 \\
\hline Overall & \multicolumn{6}{|c|}{$92.83 \pm 3.14$} & \multicolumn{6}{|c|}{$91.43 \pm 4.56$} & $\mathrm{t}=\mathbf{1 . 4 9 6}$ & 0.139 \\
\hline
\end{tabular}

Table (6) showed overall mean scores of self-care scales. Self-care maintenance scale, showed lowest percentage $(23.05 \% \pm 3.74 \%)$ during hospitalization, and improved to $44.67 \% \pm 5.84 \%$ after one month, then $44.29 \pm 7.74 \%$ after 3 months of implementing the discharge plan. Self-care management scale, was $38.43 \pm$ $3.98 \%$ during hospitalization, and improved to $64.0 \pm 6.16 \%$ and $62.0 \pm 8.15 \%$ after one and three months respectively. Self-care confidence scale, during hospitalization was $27.32 \pm 4.93 \%$ and improved to $47.34 \pm$ $8.35 \%$ and $40.83 \% \pm 11.67 \%$ after one and 3 months respectively. A statistically significant difference was noticed in relation to overall mean scores of CHF self-care scales.

Table (6): Comparison of overall mean percent scores of self-care scales among CHF studied groups during the study periods

\begin{tabular}{|c|c|c|c|c|c|c|}
\hline \multirow{3}{*}{ Heart failure self-care index } & \multicolumn{2}{|c|}{ Mean \% Score } & \multirow{2}{*}{\multicolumn{2}{|c|}{$\begin{array}{c}\begin{array}{c}\text { Correlation } \\
\text { with Baseline }\end{array} \\
\mathbf{P}\end{array}$}} & \multirow{2}{*}{\multicolumn{2}{|c|}{$\begin{array}{c}\begin{array}{c}\text { Difference from } \\
\text { Baseline }\end{array} \\
\text { Mean } \pm \text { SD. }\end{array}$}} \\
\hline & \multicolumn{2}{|c|}{ Mean \pm SD. } & & & & \\
\hline & Control group & Study group & $\begin{array}{c}\text { Control } \\
\text { group }\end{array}$ & $\begin{array}{l}\text { Study } \\
\text { group }\end{array}$ & $\begin{array}{l}\text { Control } \\
\text { group }\end{array}$ & Study group \\
\hline Self-Care Maintenance Scale: & & & & & & \\
\hline During hospitalization & $22.48 \pm 3.06$ & $23.05 \pm 3.74 \mid$ & & & & \\
\hline After one month & $23.05 \pm 3.74$ & $44.67 \pm 5.84 \mid$ & 0.350 & $<0.001^{*}$ & $0.17 \pm 1.07$ & $6.49 \pm 2.25$ \\
\hline After 3 months & $22.48 \pm 3.06$ & $44.29 \pm 7.74$ & - & $<0.001^{*}$ & 0.0 & $6.37 \pm 2.93$ \\
\hline Self-Care Management Scale: & & & & & & \\
\hline During hospitalization & $39.0 \pm 3.80$ & $38.43 \pm 3.98$ & & & & \\
\hline After one month & $38.43 \pm 3.98$ & $64.0 \pm 6.16$ & 0.586 & $<0.001^{*}$ & $0.11 \pm 1.23$ & $5.11 \pm 1.39$ \\
\hline After 3 months & $39.0 \pm 3.80$ & $62.0 \pm 8.15$ & - & $<0.001^{*}$ & 0.0 & $4.71 \pm 1.95$ \\
\hline Self-Care Confidence Scales: & & & & & & \\
\hline During hospitalization & $26.69 \pm 3.25$ & $27.32 \pm 4.93$ & & & & \\
\hline After one month & $27.32 \pm 4.93$ & $47.34 \pm 8.35$ & 0.586 & $<0.001^{*}$ & $0.11 \pm 1.23$ & $3.60 \pm 1.80$ \\
\hline After 3 months & $26.69 \pm 3.25$ & $40.83 \pm 11.67$ & - & $<0.001^{*}$ & 0.0 & $2.43 \pm 2.39$ \\
\hline
\end{tabular}

p: p-value for F test (ANOVA) with repeated measures for comparing between Baseline and each other period *: Statistically significant at $\mathrm{p} \leq 0.05$

Table (7) illustrated that the overall knowledge significantly improved patients self-care management post one month $\left(\mathrm{p}=0.033^{*}\right)$, as well improved their self-care maintenance and self-care confidence $\left(\mathrm{p}=0.001^{*}, 0.015^{*}\right.$ respectively) 3 months post implementing the discharge plan. 
Table (7): Correlation between study groups related to the three self-care scales and their five knowledge domains of self-care after one month and 3 months of follow up post implementing the discharge plan.

\begin{tabular}{|c|c|c|c|c|c|c|c|}
\hline \multirow{3}{*}{$\begin{array}{c}\text { Study group patient's five } \\
\text { knowledge domains. }\end{array}$} & & \multicolumn{6}{|c|}{ Congestive heart failure three self-care scales. } \\
\hline & & \multicolumn{3}{|c|}{1 month F/U } & \multicolumn{3}{|c|}{3 month $\mathrm{F} / \mathrm{U}$} \\
\hline & & Maintenance & Management & Confidence & Maintenanc & Management & Confidence \\
\hline \multirow{2}{*}{ Overall knowledge } & $\mathbf{R}$ & 0.217 & $0.360^{*}$ & -0.013 & $0.529^{*}$ & 0.114 & $0.407^{*}$ \\
\hline & $\mathbf{P}$ & 0.211 & $0.033^{*}$ & 0.940 & $0.001^{*}$ & 0.515 & $0.015^{*}$ \\
\hline
\end{tabular}

\section{DISCUSSION}

The results declared that in accordance with study hypotheses, an individualized nursing discharge plan, enriched with educational interventions, can significantly affect CHF adult patients' health outcomes. This is achieved through improving knowledge and promoting self-care; as those patients develop further self-confident in handling their medical responsibilities.

More than half of the study patients were between $50 \leq 60$ years old. In accordance with our results, Savarese and Lund ${ }^{(20)}$, stated that $\mathrm{HF}$ prevalence significantly increase among patients > 64 years; who comprise $<80 \%$ of HF patients. More than half of study patients were females, which was in accordance with Uchmanowicz et $\boldsymbol{a l}{ }^{(21)}$ where they reported that, approximately $50 \%$ of HF patients are women. This may be justified by the higher prevalence of hypertension, diabetes, and diastolic dysfunction among women predisposing to $\mathrm{CHF}^{(22)}$.

All studied patients complained from dyspnea, reduced exercise tolerance and fatigue, while majority reported edema, orthopnea, and paroxysmal nocturnal dyspnea. This is congruent with Ural $\boldsymbol{e t}$ $\boldsymbol{a l} \mathbf{l}^{(\mathbf{2 3})}$ who mentioned that most of CHF patients complained from peripheral edema, chest pain, and fatigue, with the clinical signs of elevated jugular venous pressure, third heart sound, increased weight, rales, orthopnea or paroxysmal nocturnal dyspnea (23). This signifies the importance of individualized nursing interventions in order to decrease readmission rates. Cowie et al. $^{(24)}$ found that; CHF patient's deterioration is accompanied by fluid retention signs known as extended jugular vein, edema, lung congestion, ascites and third heart sound, which are necessitating urgent medical attention.

In addition, the majority of patients had IHD, while, less than two-thirds had hypertension, diabetes mellitus, and anemia. In this regard, Mesquita et al. (19) pointed that; many of HF patients have multiple either cardiac or extra-cardiac co-morbidities, increasing re-hospitalization risk. Moreover, Madelaire et al. ${ }^{(25)}$ found that; the noncardiovascular causes which were often reported by hospitalized HF patients, signifies multidisciplinary approach in order to reduce re-hospitalizations.

More than one-quarter of our patients were diagnosed with $\mathrm{HF}$ for three to $\leq$ five years. In this context, Heart Disease and Stroke Statistics ${ }^{(26)}$ reported that; about half of HF patients live beyond 5 years after being diagnosed. However, there is no clear average life expectancy. This could be explained by the fact that CHF is not curable, but early detection and treatment plan compliance may improve QOL, which in-turn leads to increased life expectancy. In addition, Akita et $\boldsymbol{a l} .{ }^{(27)}$ emphasized the importance of monitoring HF patients after discharge for improving their clinical outcomes being at higher risk of readmission and death within three years.

Dietary intake of both groups was not consistent with recommended regimen. Researchers found that HF patients' adherence to low sodium diet is difficult without continuous family support, which contributes to worsening symptoms and increases rehospitalization ${ }^{(28)}$.

A statistically significant difference between both groups was observed regarding follow up commitment. This could be attributed to increased patients' awareness about the importance of follow up and prognosis. This goes in-line with McAlister et al. ${ }^{(29)}$ who found that; early scheduled outpatient follow-up appointments are associated with better outcomes within 30 days after discharge to prevent readmissions.

Chemistry tests, ECG and echocardiography had been requested for all studied patients. This could be explained by that CHF confirmation is determined by routine examinations as valuable information. Inamdar and Inamdar ${ }^{(30)}$ stated that routine biochemical analysis and echocardiography should be performed on admission for reliable clinical diagnosis to estimate CHF severity, and deciding suitable treatment.

All studied patients had reduced cardiac output, excess fluid volume, activity intolerance and knowledge deficit, while more than half had impaired gas exchange. This goes with a study illustrating that most frequent hospitalized CHF patients nursing diagnoses were; reduced cardiac 
output, excessive fluid volume, activity intolerance, impaired gas exchange, deficient of knowledge, and impaired physical mobility ${ }^{(31)}$.

The overall CHF knowledge mean percent scores denoted a statistically significant difference between both studied groups explaining better knowledge among study group. Studies recommend implementing a comprehensive individualized CHF nursing discharge planning with continuous monitoring in the outpatient clinic to enhance knowledge, reduce readmissions, improve QOL and increase self-care adherence ${ }^{(32,33)}$.

There was no statistically significant difference concerning overall self-care of heart failure index mean percent among control group. This could be explained by the absence of educational programs, which resulted in non-compliance with self-care practices. In this respect, Tovar ${ }^{(33)}$ illustrated that self-care activity commitment is based on patients' knowledge for making symmetrical choices with provided medical regimen.

Findings also showed a highly statistically significant difference regarding overall mean percent scores of HF self-care index among study group after 1 and 3 months of implementing the discharge plan. In our opinion may be due to decreasing patient's retention of information with time.

Similar studies reported that; the application of a standardized educational nursing intervention for CHF patients reflects improvement of self-care management, self-care behaviors and strongly change self-care scale scores in those HF patients. Moreover, enhances self-confidence to perform optimal self-care management and maintenance, to reduce clinical symptom burden ${ }^{(34,35)}$.

\section{CONCLUSION}

The current study proved that, CHF patients who received integrated discharge plan with educational program exhibit healthy respiratory and cardiac outcomes in addition to better functional abilities than those who did not. Furthermore, overall knowledge was also improved and significantly reflected in self-care index practices after 1 and 3 months of implementing the discharge plan. Thus, adequate education is systematically associated with higher HF related knowledge.

\section{REFERENCES}

1. Mayo Clinic Staff (2018): Heart disease care at Mayo Clinic. https://www.mayoclinic.org/diseasesconditions/heart-disease/care-at-mayo-clinic/mac20353128

2. American Heart Association (2017): Heart failure projected to increase dramatically, according to new statistics. 7272, Dallas. https://www.heart.org /en/news/ 2018/05/01/heart-failure-projected-toincrease-dramatically-according-to-new-statistics

3. Ponikowski P, Voors A, Anker S et al. (2016): ESC Guidelines for the diagnosis and treatment of acute and chronic heart failure: The Task Force for the diagnosis and treatment of acute and chronic heart failure of the European Society of Cardiology (ESC).Developed with the special contribution of the Heart Failure Association (HFA) of the ESC. European Heart Journal, 37 (27): 2129-2200.

4. Cummings E, Showell C, Roehrer E et al. (2010): Discharge, Referral and Admission: A Structured Evidence-based Literature Review, eHealth Services Research Group, University of Tasmania, Australia (on behalf of the Australian Commission on Safety and Quality in Health Care, and the NSW Department of Health. https://www.safetyandquality. gov.au/sites/default/files/migrated/Dischargeadmission-and-referral-literature-review-FINAL-5October-2010.pdf

5. Amakali K (2015): Clinical Care for the Patient with Heart Failure: A Nursing Care Perspective. Cardiovascular Pharmacology, 4 (2): 2-5.

6. Colet J, Enjuanes C, Lupónd J et al. (2016): Transitions of Care Between Acute and Chronic Heart Failure: Critical Steps in the Design of a Multidisciplinary Care Model for the Prevention of Rehospitalization. Revista Espanola de Cardiologia, 69 (10): 951-961.

7. Riegel B, Moser D, Buck H et al. (2017): Self Care for the Prevention and Management of Cardiovascular Disease and Stroke. A Scientific Statement for Healthcare Professionals from the American Heart Association. J Am Heart Assoc., 6 (9): 6997-4.

8. Mcmurray J, Adamopoulos S, Anker S et al. (2012): ESC Guidelines for the diagnosis and treatment of acute and chronic heart failure. European Journal of Heart Failure, 14 (8): 803-869.

9. Qoli A, Nasrabadi T, Abianeh E (2016): The Effect of an Educational Self-Care Program on Knowledge and Performance in Patients with Coronary syndrome. International Journal of Medical Research \& Health Sciences, 5: 132-137.

10. Jurgens C, Goodlin S, Dolansky M et al. (2015): Heart Failure Management in Skilled Nursing Facilities: Guidelines.A Scientific Statement From the American Heart Association and the HeartFailure Society of America. Journal of Cardiac Failure, 21 (4): 6-22.

11. Garde A, Sörnmo L, Jané $R$ et al. (2010): Breathing Pattern Characterization in Chronic Heart Failure Patients Using the Respiratory Flow Signal. Ann Biomed Eng., 38 (12): 3572- 3580.

12. Reilly C, Higgins $M$, Rebecca A et al. (2009): Development, Psychometric Testing, and Revision of the Atlanta Heart Failure Knowledge Test. Journal of Cardiovascular Nursing, 24 (6): 500-509.

13. Butts B, Higgins M, Dunbar $S$ et al. (2018): The Third Time's A Charm: Psychometric Testing and 
Update of the Atlanta Heart Failure Knowledge Test. Journal of Cardiovascular Nursing, 33 (1): 13-21.

14. Zaharias E, Cataldo J, Mackin L et al. (2014): Simple Measures of Function and Symptoms in Hospitalized Heart Failure Patients Predict ShortTerm Cardiac Event-Free Survival. Nursing Research and Practice, 1: 815984-92.

15. Cassel J (2014): Palliative Care's Impact on Utilization and Costs: Implications for Health Services Research and Policy. In: Kelley AS, Meier DE, editors. Meeting the Needs of Older Adults with Serious Illness: Challenges and Opportunities in the Age of Health Care Reform. New York, Springer Science Business Media. Pp: 109-126. https://cdn.mdedge.com/files/s3fs-

public/Document/April2018/jhm_issue_0318_final.pdf

16. Ibrahim A, Tawfik F, Abudari M (2016): Using a self-care management tool to improve self-care and quality of life in patients with congestive heart failure. Clinical Nursing Studies, 4 (3): 3-11.

17. Riegel B, Lee S, Dickson V et al. (2009): An Update on the Self-Care of Heart Failure Index. J Cardiovasc Nurs., 24 (6): 485-497.

18. Siabani S, Stephen $R$, Patricia $M$ et al. (2014):Translation and Validation of the Self-care of Heart Failure Index into Persian. Journal of Cardiovascular Nursing, 29 (6): 1-5.

19. Mesquita E, Jorge A, Rabelo L et al. (2017): Understanding Hospitalization in Patients with Heart Failure. International Journal of Cardiovascular Sciences, 30 (1): 81-90.

20. Savarese G, Lund L (2017): Global Public Health Burden of Heart Failure. Cardiac Failure Review, 3 (1): 7-11.

21. Uchmanowicz I, Nessler J, Gobbens $\mathbf{R}$ et al. (2019): Coexisting Frailty With Heart Failure. Front Physiol., 10: 791-95.

22. Bozkurt B, Khalaf S (2017): Heart Failure in Women. Methodist Debakey Cardiovascular Journal, 13 (4): 216-223.

23. Ural D, Çavuşoğlu Y, Eren M et al. (2016): Diagnosis and management of acute heart failure. Anatol Journal of Cardiology, 15 (11): 860-889.

24. Cowie M, Anker S, Cleland J et al. (2015): Improving care for patients with acute heart failure: before, during and after hospitalization. ESC Heart Failure, 1: 110-145
25. Madelaire C, Gustafsson $\mathbf{F}$, Kristensen $\mathrm{S}$ et al. (2019): Burden and Causes of Hospital Admissions in Heart Failure During the Last Year of Life. JACC: Heart Failure, 7 (7): 561-570.

26. Heart Disease and Stroke Statistics. (2016): A Report From the American Heart Association. Retrieved from https://ahajournals.org/doi/full/ 10.1161/cir.0000000000000350

27. Akita K, Kohno T, Kohsaka S et al. (2019): Prognostic Impact of Previous Hospitalization in Acute Heart Failure Patients. The Japanese Circulation Society, 83 (6): 1261-1268.

28. Chung M, Lennie T, Martin G et al. (2015): Adherence to the Low Sodium Diet in Patients with Heart Failure is Best When Family Members also Follow the Diet: A Multicenter Observational Study. Journal of Cardiovascular Nursing, 30 (1): 44-50.

29. McAlister F, Youngson E, Kaul P et al. (2016): Early Follow-Up After a Heart Failure Exacerbation: The Importance of Continuity. Circulation: Heart Failure, 9 (9): e003194.

30. Inamdar A, Inamdar A (2016): Heart Failure: Diagnosis, Management and Utilization. Journal of Clinical Medicine, 5 (7): 62-65.

31. Cavalcanti A, Pereira J (2014): Nursing diagnoses of patients with heart failure: an integrative review. Online Brazilian Journal of Nursing, 13 (1): 113-125.

32. Mazloum S, Gorji M, Gholkhatmi M et al. (2016): Effectiveness of discharge-planning on physical quality of life of patients with ischemic heart disease. International Journal of Applied Basic Medical Research, 6 (2): 129-133.

33. Tovar N (2016): The Effects of Patient Education and Knowledge of CHF Patients As Evaluated by the PakSAC Survey. The Eleanor Mann School of Nursing Undergraduate Honors Theses. Retrieved from https://scholarworks.uark.edu/nursuht/49

34. Gázquez M, Holguín E, Cortés R (2012): Effectiveness of an educational program in nursing in the self-care of patients with heart failure: randomized controlled trial. Revista LatinoAmericana de Enfermagem, 20 (2): 296-306.

35. McGreal M, Hogan $M$, Walsh-Irwin $C$ et al. (2014): Heart failure self-care interventions to reduce clinical events and symptom burden. Research Reports in Clinical Cardiology, 5: 243- 257. 